\title{
The feasibility and positive effects of a customised videogame rehabilitation programme for freezing of gait and falls in Parkinson's disease patients: a pilot study
}

Dijana Nuic ${ }^{1,2}$, Maria Vinti ${ }^{2}$, Carine Karachi ${ }^{1,3}$, Pierre Foulon ${ }^{2,4}$, Angèle Van Hamme ${ }^{1,5}$ and Marie-Laure Welter ${ }^{1,2,5,6^{*}}$ (1)

\begin{abstract}
Background: Freezing of gait and falls represent a major burden in patients with advanced forms of Parkinson's disease (PD). These axial motor signs are not fully alleviated by drug treatment or deep-brain stimulation. Recently, virtual reality has emerged as a rehabilitation option for these patients. In this pilot study, we aim to determine the feasibility and acceptability of rehabilitation with a customised videogame to treat gait and balance disorders in PD patients, and assess its effects on these disabling motor signs.
\end{abstract}

Methods: We developed a customised videogame displayed on a screen using the Kinect system. To play, the patient had to perform large amplitude and fast movements of all four limbs, pelvis and trunk, in response to visual and auditory cueing, to displace an avatar to collect coins and avoid obstacles to gain points. We tested ten patients with advanced forms of PD (median disease duration = 16.5 years) suffering from freezing of gait and/or falls (Hoehn\&Yahr score $\geq 3$ ) resistant to antiparkinsonian treatment and deep brain stimulation. Patients performed 18 training sessions during a $6-$ 9 week period. We measured the feasibility and acceptability of our rehabilitation programme and its effects on parkinsonian disability, gait and balance disorders (with clinical scales and kinematics recordings), positive and negative affects, and quality of life, after the 9th and 18th training sessions and 3 months later.

Results: All patients completed the 18 training sessions with high feasibility, acceptability and satisfaction scores. After training, the freezing-of-gait questionnaire, gait-and-balance scale and axial score significantly decreased by 39, 38 and $41 \%$, respectively, and the activity-balance confidence scale increased by $35 \%$. Kinematic gait parameters also significantly improved with increased step length and gait velocity and decreased double-stance time. Three months after the final session, no significant change persisted except decreased axial score and increased step length and velocity.

Conclusions: This study suggests that rehabilitation with a customised videogame to treat gait and balance disorders is feasible, well accepted, and effective in parkinsonian patients. These data serve as preliminary evidence for further larger and controlled studies to propose this customised videogame rehabilitation programme at home.

Trial registration: ClinicalTrials.gov NCT02469350.

Keywords: Parkinson's disease, Freezing of gait, Falls, Videogame, Rehabilitation

\footnotetext{
* Correspondence: marielaure.welter@icm-institute.org

${ }^{1}$ CNRS, UMR7225, Institut du Cerveau et de la Moelle Epinière, Sorbonne universités, Université Pierre et Marie Curie (UPMC) Paris P6; UMRS 1127, 75013 Paris, France

2LabCom BRAIN e-NOVATION, Institut du Cerveau et de la Moelle épinière

(ICM), 75013 Paris, France

Full list of author information is available at the end of the article
}

(c) The Author(s). 2018 Open Access This article is distributed under the terms of the Creative Commons Attribution 4.0 International License (http://creativecommons.org/licenses/by/4.0/), which permits unrestricted use, distribution, and reproduction in any medium, provided you give appropriate credit to the original author(s) and the source, provide a link to the Creative Commons license, and indicate if changes were made. The Creative Commons Public Domain Dedication waiver (http://creativecommons.org/publicdomain/zero/1.0/) applies to the data made available in this article, unless otherwise stated. 


\section{Background}

Parkinson's disease (PD) is the second most common neurodegenerative disorder resulting mainly from the loss of dopaminergic midbrain neurons. Clinically, this disease is characterised by akinesia, with motor slowness, decreased amplitude of automatic movements and movement initiation failure, rigidity and tremor [1], and is improved by dopamine replacement therapy [2]. Patients with PD also present non-motor signs with dysexecutive syndrome, apathy, anxiety and depression [3]. Up to now, no treatment exists to arrest or slow down the neurodegenerative process. In advanced and severe forms, freezing of gait (FOG) and postural instability with falls occur in about $50-75 \%$ of patients [4]. These signs worsen with time and become unresponsive to drug treatment or deep-brain stimulation [5], leading to increased mortality and morbidity, poor life quality and high healthcare costs [6].

In PD patients, physiotherapy represents an additional treatment to maximise functionality through movement rehabilitation. It focuses on transfers, posture, upper limb function, balance, gait, and physical activity, also including cueing strategies, cognitive movement strategies, and exercise [7]. Various techniques have been proposed within physiotherapy with general physiotherapy, exercise, treadmill walking, cueing (with auditory, visual or somatosensory cues), dance or martial arts, with short-term improvements of gait speed, freezing of gait, balance and falls, global motor function, activities of daily living, and quality of life [7], with no clear evidence of differences in the short-term effects of treatment between physiotherapy methods [7, 8], but a better outcome if balance dysfunction is addressed [8]. A recent meta-analysis highlights the long-term positive effects of physical therapy with improved gait and balance performance, enhanced activities of daily living and quality of life, in particular for progressive resistance training, as long as it is maintained over time [9]. Finally, the majority of studies addressing the effects of physiotherapy and exercise have revealed a loss of benefit within weeks or months after discontinuation of training [8]. Moreover, several barriers for exercise compliance have been identified in people with PD with low perceived benefit of exercise, lack of time, as well as fear of falling [10]. The use of virtual reality has therefore emerged as a promising rehabilitation option for PD with the possibility of improving long-term adherence to exercise in a personalised, motivating and engaging manner [11]. Recent studies have suggested that virtual reality training, such as combined with treadmill walking $[12,13]$ or cycling [14], produces short-term benefits on gait, balance, activities of daily living, cognition, quality of life, with good exercise adherence and no adverse events [15]. Treadmill walking coupled with virtual reality also promotes fall reduction 6 months after the cessation of training [13]. Virtual reality exercises stimulate movement, optimise motor learning, potentially compensate defective internal mechanisms using external cueing and allow augmented feedback [14]. In addition to a challenging and motivating virtual environment, gaming also allows training for specific motor and non-motor signs with an amusing and enjoyable approach, that can be performed at home with increased training frequency and/or on demand [16]. A few studies have tested the effects of commercial games using Nintendo $\mathrm{Wii}^{\mathrm{TM}}$ or Xbox Kinect ${ }^{\mathrm{Tm}}$ systems in PD patients and reported good feasibility [17] with increased balance [18-20] and activities of daily living $[21,22]$ performance, mood and quality of life [22]. In some of these previous studies, the effects of commercial games were not specific to the disease with similar changes in healthy controls after training $[18,23]$. These PD patients also only suffered from mild to moderate forms of PD, with no disabling or refractory gait and balance disorders. Lastly, training with commercial games may not be suitable for such PD patients due to their specific motor disability [17]. One customised game has recently been tested for arm movement training in 7 PD patients with encouraging results [24].

Here, we propose a virtual reality training system using a customised game specifically designed to treat refractory gait and balance disorders of PD patients according to recommended physiotherapy programmes for PD patients with high amplitude and fast movements, combined with visual and auditory cueing [25]. The aim of this pilot study was to assess the feasibility and acceptability of our customised videogame in 10 PD patients with FOG and/or postural instability with falls resistant to dopaminergic treatment and deep brain stimulation. We examined the effects of our game training on gait and balance control by using both clinical and kinematic gait recordings, with a final examination of the patients 3 months after the last session in order to assess the persistence of training benefits.

\section{Methods \\ Study design}

This study was a pilot study. Eligible participants consisted of PD patients. This study is part of clinical trial C15-12 sponsored by Inserm (ID RCB: 2015-A00277-42). It was granted approval by local Ethics Committee (Comité de protection des personnes-Ile de France V on June 2, 2015), authorised by the French authorities (ANSM, 150358B-31), and registered in a public trials registry (Trial Registration: ClinicalTrials.gov NCT02469350). All study participants gave their informed, written consent to participation, in line with French ethical guidelines.

\section{The 'Toap run' videogame}

The videogame ('Toap Run') was built specifically for this study. The videogame was controlled by a laptop (Asus ${ }^{\oplus}$, Model G551 J, Intel ${ }^{\oplus}$ Core $^{\mathrm{Tm}}$ i7, NVIDIA ${ }^{\oplus}$ GEFORCE $^{\oplus}$ GTX 
TM8, 8 GB of RAM) and displayed on a high definition television screen (Phillips, 50-in. plasma, resolution 1920 by 1080 pixels) placed $2 \mathrm{~m}$ in front of the patient. The patient played by moving in front of a RGB-D (Red Green Blue + Depth) Kinect ${ }^{\text {tix }}$ motion sensor (Version 2, Microsoft, USA) placed below the screen.

The scenario consists of a small animal (the avatar) that moves around three different environments: a garden, a mine cart and a surfboard on a lake (Fig. 1). To play, the patient, standing upright in front of the screen, has to move to induce displacements of the avatar in real time within these different environments, to collect coins and avoid obstacles to gain points, in relationship to visual and auditory cues. The movements consist of large amplitude rapid movements of all four limbs, pelvis and trunk, with lateral, vertical and forward displacements

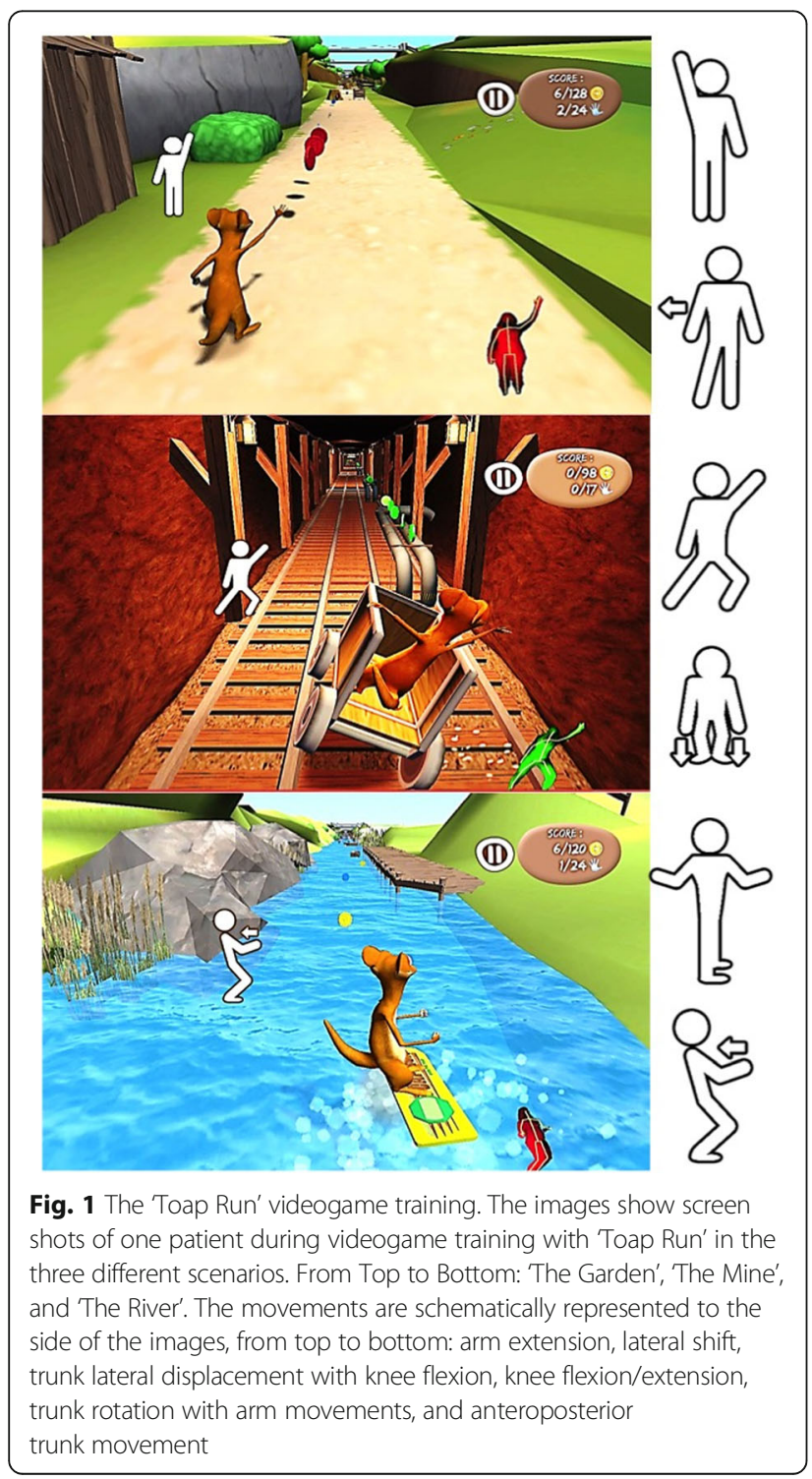

of the legs, to reinforce foot lifting and postural control (Fig. 1):

- In 'The Garden', the patients had to extend their arms to collect coins and perform lateral displacements to avoid laterally placed obstacles,

- In 'The Mine', the patients had to collect coins and avoid obstacles with the same movements as in 'The Garden', plus knee flexion/extension to avoid high level obstacles and lateral trunk displacements with one leg flexion to move the wagon and collect coins,

- In 'The River', the patients had to perform trunk rotation and arm movements to collect coins, and anteroposterior trunk movements to avoid obstacles.

Visual cueing consisted of a schematic representation of the movements required to perform along the trajectory of the avatar. Auditory cueing consisted of rhythmic music that beats in relationship to the velocity of the avatar displacement. Three different rhythms were presented: 20, 30 and 40 beats $/ \mathrm{min}$. The first session was stereotyped and consisted of a 15 min session with a 20 beat velocity first in the garden and then in the mine, corresponding to a total of 100-150 movements. Game difficulty, i.e. velocity and number of movements to capture the coins and avoid obstacles, was then adjusted manually and individually by the physiotherapist for subsequent rehabilitation sessions, in relation to the patient's ability. For this purpose, the physiotherapist was instructed to increase the rhythm, number and complexity of movements, i.e. the environment, depending on the performance in the previous session with a view to obtain a session of at least 40 min duration after the 9th session [26].

\section{Participants}

Between June 2015 and May 2016, ten people with PD (mean age: $64.2 \pm 6.1$ years, $5 \mathrm{~F} / 5 \mathrm{M}$ ) were recruited at the Pitié-Salpêtrière Hospital and included according to the following criteria: 1) diagnosis of $\mathrm{PD}, 2$ ) age below 71 years, 3) disabling FOG and/or falls despite optimal drug treatment and subthalamic deep-brain stimulation (items 13-falls and/or item 14-FOG of the UPDRS part II $\geq 2$ ON treatment) [27] (Table 1). Exclusion criteria included dementia (Mini Mental State Examination Score $<24$ ), inability to maintain upright posture or walk independently, patients suffering from other pathologies affecting gait and balance, or severe medical conditions that prevented assessment.

\section{Procedure}

The videogame rehabilitation programme consisted of 18 training sessions with the 'Toap Run' videogame over a period of 6 weeks, in addition to the usual anti-parkinsonian 
Table 1 Clinical and demographic features of PD patients at inclusion

\begin{tabular}{|c|c|c|c|c|c|c|c|c|c|c|}
\hline $\mathrm{N}^{\circ}$ patient & $\begin{array}{l}\text { Sex/Age } \\
\text { (yrs) }\end{array}$ & $\begin{array}{l}\text { Disease } \\
\text { duration } \\
\text { (yrs) }\end{array}$ & $\begin{array}{l}\text { Time since } \\
\text { STN-DBS } \\
\text { (months) }\end{array}$ & $\begin{array}{l}\text { Falls/FOG } \\
\text { duration } \\
\text { (months) }\end{array}$ & $\begin{array}{l}\text { UPDRS part II: } \\
\text { falls/FOG }\end{array}$ & $\begin{array}{l}\text { UPDRS } \\
\text { part III } \\
(/ 108)\end{array}$ & $\begin{array}{l}\text { Hoehn\&Yahr } \\
\text { stage (/5) }\end{array}$ & $\begin{array}{l}\text { FOG-Q } \\
(/ 64)\end{array}$ & $\begin{array}{l}\text { ABC } \\
\text { scale (\%) }\end{array}$ & $\begin{array}{l}\text { LEED } \\
\text { (mg/day) }\end{array}$ \\
\hline 01 & $M / 58$ & 13 & 26 & 20 & $2 / 3$ & 21 & 3 & 32 & 70 & 700 \\
\hline 02 & $F / 68$ & 21 & 52 & 14 & $3 / 2$ & 27 & 4 & 32 & 39 & 700 \\
\hline 03 & $M / 62$ & 15 & 60 & 30 & $2 / 3$ & 20 & 3 & 22 & 51 & 350 \\
\hline 04 & $F / 69$ & 16 & 93 & 80 & $2 / 2$ & 13 & 3 & 27 & 46 & 300 \\
\hline 05 & $\mathrm{M} / 70$ & 17 & 18 & 13 & $2 / 3$ & 15 & 3 & 21 & 69 & 600 \\
\hline 06 & M/69 & 32 & 183 & 159 & $4 / 4$ & 32 & 4 & 30 & 50 & 200 \\
\hline 07 & $F / 69$ & 20 & 34 & 26 & $2 / 3$ & 13 & 3 & 20 & 22 & 600 \\
\hline 08 & $F / 61$ & 13 & 37 & 25 & $2 / 2$ & 14 & 3 & 17 & 64 & 400 \\
\hline 09 & $F / 53$ & 23 & 140 & 65 & $2 / 3$ & 15 & 3 & 30 & 31 & 300 \\
\hline 10 & $M / 61$ & 11 & 20 & 18 & $2 / 3$ & 33 & 4 & 28 & 72 & 700 \\
\hline Mean (SD) & $64.0(5.8)$ & $18.1(6.2)$ & $66.3(55.8)$ & $45.0(45.9)$ & $\begin{array}{l}2.3(0.7) \\
/ 2.8(0.6)\end{array}$ & $20.3(7.8)$ & $3.3(0.5)$ & $25.9(5.4)$ & $51.4(17.3)$ & 485 (205) \\
\hline
\end{tabular}

$A B C$ activity balance confidence, FOG-Q freezing of gait questionnaire, $L E E D$ levodopa-equivalent dosage, STN-DBS subthalamic deep brain stimulation, UPDRS unified Parkinson's disease rating scale

treatment, which was unchanged throughout the duration of the protocol. Training was performed in the institute and supervised by a physiotherapist (DN).

Clinical assessments and kinematic parameters of gait and balance disorder recordings were performed at inclusion (before the first training session), after the 9th and 18th training sessions, and 3 months later.

\section{Gait initiation recordings}

The gait initiation process was specifically studied as this requires simultaneous forward movement (locomotion) and balance control, in order to maintain stability and prevent falling [28]. Kinematic parameters of gait initiation were recorded using a force platform $(0.9 \times 1.8 \mathrm{~m}$, AMT Inc. LG6-4-1). Subjects, barefoot and standing upright, were instructed to commence walking for $5 \mathrm{~m}$ following an auditory cue. Ten consecutive trials were recorded, the subject being instructed to walk at a selfpaced speed [29].

\section{Outcome measures}

The primary outcome measure was the feasibility of the videogame rehabilitation programme using a Likert-scale (1 to 7 points per item) specific questionnaire [30]. With this scale we measured the perceived interest (4 items, with scores ranging from 4 to 28 , higher scores indicating better perceived interest), perceived competence ( 8 items, with scores ranging from 8 to 56, higher scores indicating higher perceived competence) and perceived difficulty (3 items, with scores ranging from 3 to 21, lower scores indicating lower perceived difficulty). This questionnaire was performed after the first training and once a week.
Secondary outcomes included the acceptability scale evaluating the level of interest, satisfaction and discomfort in the game by using a visual analogue scale ranging from zero to 10 . Perceived fatigue was evaluated by using the 'sensory' and 'cognition' subscales of the Piper Fatigue Revised Scale (with scores ranging from 0 to 10 , higher scores indicating higher perceived fatigue) [31], and perceived affects using the Positive and Negative Emotionality questionnaire (EPN-31) that comprised 31 items quantifying the frequency of each affect (7-point scale, ranging from 1 "not experienced at all" to 7 "experienced this affect several times each day") with 3 subscores: positive, negative and surprise affects [32]. The users' experience in the game was assessed using a 5-point Likert scale. Game performance (percentage of coins captured), number of movements and duration of each training session were also recorded. These questionnaires were administered after the first session and once a week.

Other secondary outcomes focused on gait and balance disorders including the parkinsonian motor disability (UPDRS part III, range $=0$ to 108) [27], 'axial' (sum of items 28-29-30-31 of the UPDRS part III, range $=0$ to 16$)$, gait and balance scale (GABS part Bclinical examination, range $=0$ to 38) [33], FOG questionnaire (FOG-Q, range $=0$ to 64) [34], fear of falling (Activity Balance Confidence-ABC, range $=0$ to $100 \%$ ) [35], activities of daily living (ADL-UPDRS part II, range $=0$ to 42) [27] and quality of life (Summary index of the Parkinson's disease Questionnaire-39: SIPDQ39, range $=0-100 \%$ ) [36] scales. Higher scores in these scales indicate greater disability and/or more 
severe signs, except the $A B C$ scale for which higher scores indicate higher confidence. The fall frequency was assessed with item 12 of the FOG-Q: 'How often do you fall?' with scores ranging from 0 to $4(0=$ Never, $1=$ Very rarely-about once a year, $2=$ rarelyabout once a month, $3=$ often- about once a week, 4 = very often-once a day or more) [34].

Gait initiation kinematics parameters were the anteroposterior and lateral displacements of the centre of foot pressure (CoP) during the anticipatory postural adjustments (APAs) phase, length and velocity of the first step and braking index (reflecting active postural control) $[29,37]$ (Fig. 2). The duration of the APAs phase (time between the first biomechanical event and foot-off of the swing foot) and double stance time (time between the foot-contact of the swing foot and foot-off of the stance foot) were also measured (Fig. 2). Clinical assessments and gait recordings were performed at baseline (inclusion), after the 9th and 18th sessions, and 3 months later, in the morning with usual antiparkinsonian treatment and stimulation parameters.

\section{Statistical analysis}

To assess the changes in the feasibility, acceptability, perceived fatigue, emotion questionnaires, motivation, movements during gaming and game duration, we first tested the data distribution using the ShapiroWilk test and the variance homogeneity using Levene's test. If data were found to be normally distributed and show homogeneity, we performed repeated measures ANOVAs, and in case of non-normal distribution, non-parametric Friedman's ANOVAs. If the results were significant, Tukey's post-hoc tests were performed for parametric data, and Nemenyi post-hoc tests for Friedman's ANOVA.

The changes in the clinical scores between baseline and after the 9th and 18th sessions, and 3 months later were assessed using the non-parametric Wilcoxon tests. We used the MacNemar test to measure the change in fall frequency between baseline and after the 18th session.

Lastly, the changes in the kinematic gait parameters were measured using a linear mixed model.

All statistical analyses were performed using the $\mathrm{R}$ package lme4 (version 1.1-12, R-studio Inc., R Core Development Team). Results were considered significant at $p<0.05$.

\section{Results}

\section{Game training sessions}

All patients performed the 18 training sessions. Game duration, game difficulty (with increased visual flow of the virtual environment and number of

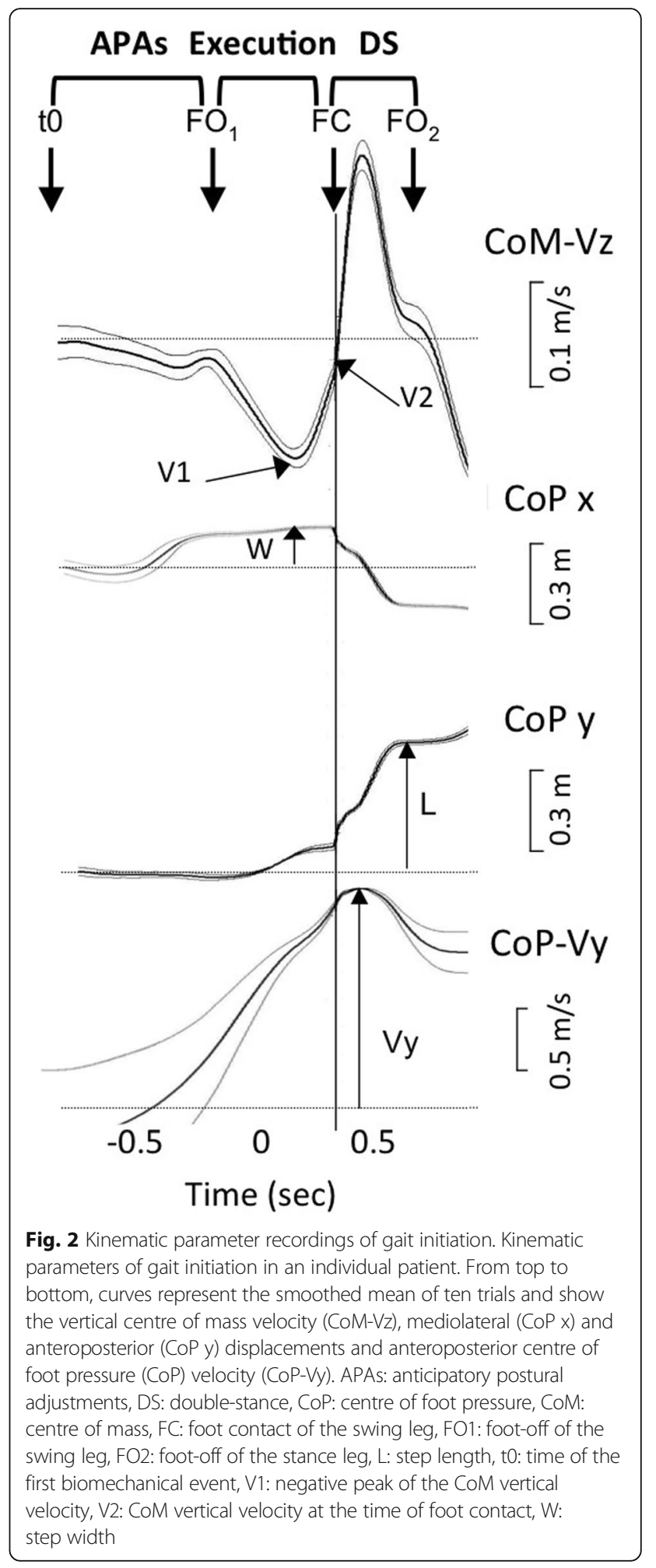

movements to perform) and game performance increased significantly over time (Friedman's ANOVA; Game duration: $34.0 \pm 10.3 \mathrm{~min}, \chi^{2}$ [17] $=102.3$, $p<10^{-4}$; Number of movements: $654.2 \pm 351.9, \mathrm{x}^{2}$ 
[17] $=120.9, p<10^{-4}$; Game performance: $76.3 \% \pm$ $14.5 \%, X^{2}[17]=32.3, p=0.01$, Fig. 3, Table 2). Post-hoc analysis revealed that game duration increased significantly between the first and 8th session and those following, the 2nd and 10th session and those following, the 3rd and the 12th session and those following and between the 4th, 5th and 6th sessions and the 16th to the 18th session (Table 2). Similarly, the number of movements significantly increased between both the 1st and 2nd sessions and the 10th and those following, and between the 3rd, 4th, 5th and 6th sessions and the 14th to the 18th session (Table 2). Lastly, post-hoc analysis revealed that the game performance had

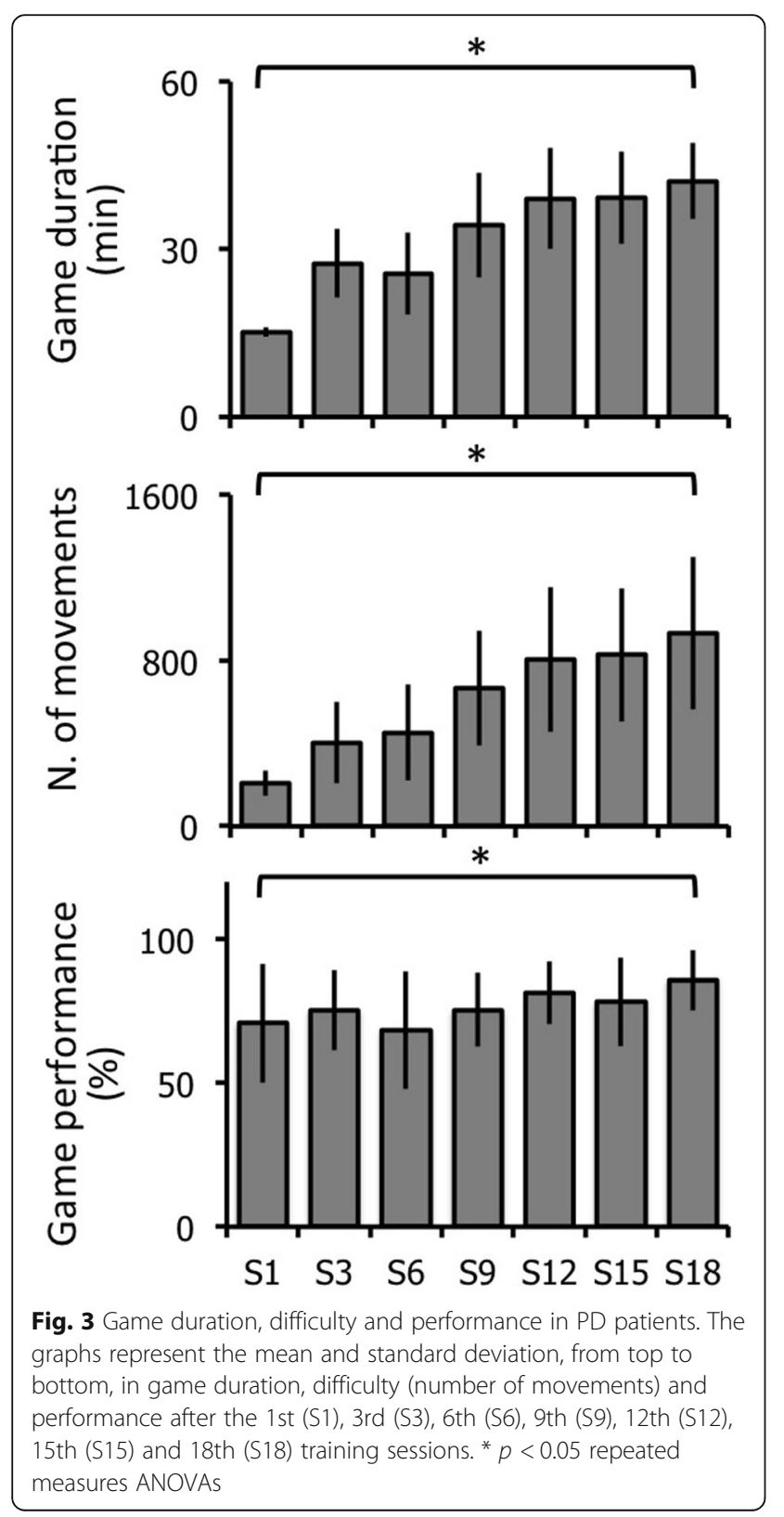

Table 2 Number of movements, game duration and performance of PD patients over training sessions

\begin{tabular}{|c|c|c|c|}
\hline $\mathrm{N}^{0}$ session & $\begin{array}{l}\text { Game } \\
\text { duration (min) }\end{array}$ & Number of movements & $\begin{array}{l}\text { Game } \\
\text { performance (\%) }\end{array}$ \\
\hline 01 & $15.1(0.8)$ & $207(61)$ & $70.8(20.8)$ \\
\hline 02 & $22.0(6.2)$ & $323(120)$ & $72.6(15.9)$ \\
\hline 03 & $27.5(6.2)$ & $403(195)$ & $75.3(13.9)$ \\
\hline 04 & $28.9(2.9)$ & $448(165)$ & $75.6(16.6)$ \\
\hline 05 & $26.7(6.1)$ & $440(246)$ & $71.5(17.6)$ \\
\hline 06 & $25.7(7.4)$ & $452(230)$ & $68.4(20.6)$ \\
\hline 07 & $33.8(4.6)$ & $615(270)$ & $79.5(12.6)$ \\
\hline 08 & $36.7(5.5)^{\mathrm{a}}$ & $626(154)$ & $76.4(13.4)$ \\
\hline 09 & $34.4(9.4)^{\mathrm{a}}$ & $666(280)$ & $75.4(12.9)$ \\
\hline 10 & $40.4(10.2)^{a, b}$ & $813(380)^{a, b}$ & $74.3(9.1)$ \\
\hline 11 & $38.5(7.1)^{a, b}$ & $785(241)^{a, b}$ & $77.0(12.6)$ \\
\hline 12 & $39.1(9.1)^{a, b, c}$ & $806(351)^{a, b}$ & $75.0(18.1)$ \\
\hline 13 & $37.9(6.0)^{\mathrm{a}}$ & $744(285)^{a, b}$ & $81.5(10.9)$ \\
\hline 14 & $39.8(4.4)^{a, b, c, f, f}$ & $860(354)^{a, b, c, d, d, e, f}$ & $76.0(11.2)$ \\
\hline 15 & $39.2(8.3)^{a, b, c}$ & $827(322)^{a, b, c, c, d, e f}$ & $79.5(12.8)$ \\
\hline 16 & $41.6(12.1)^{a, b, c, c, d, e, f}$ & $899(438)^{a, b, c, c, d, e, f}$ & $78.2(15.4)$ \\
\hline 17 & $42.9(7.6)^{a, b, c, d, e, f}$ & $931(410)^{a, b, c, d, d, e, f}$ & $81.9(11.9)$ \\
\hline 18 & $42.2(6.8)^{a, b, c, d, d, f, f}$ & $932(368)^{a, b, c, c, d, e, f}$ & $85.8(10.5)^{f}$ \\
\hline
\end{tabular}

Values are mean (SD)

${ }^{a} p<0.05$ as compared to Session 01

${ }^{\mathrm{b}} p<0.05$ as compared to Session 02

${ }^{c} p<0.05$ as compared to Session 03

${ }^{d} p<0.05$ as compared to Session 04

${ }^{\mathrm{e}} p<0.05$ as compared to Session 05

${ }^{\mathrm{f}} p<0.05$ as compared to Session 06

increased significantly between the 6th and 18th sessions (Table 2).

Feasibility, acceptability and emotional perception Patients reported high-perceived interest and competence scores for videogame training, these scores were stable over time (Friedman's ANOVA; Interest: $25.4 \pm$ 3.4; $\chi^{2}[5]=10.6, p=0.06$; ANOVA; competence: 41.3 \pm 9.1 ; F $[5,45]=0.92, p=0.47$, Fig. 4). Perceived difficulty was low and also stable over time (ANOVA, $11.85 \pm 3.4 ; \mathrm{F}[5,45]=0.36, p=0.87$, Fig. 4). General acceptability increased significantly over time (Friedman's ANOVA, 8.39 $\pm 1.56 ; x^{2}[5]=20.5, p=$ $10^{-4}$, Fig. 4). Post-hoc analysis revealed that general acceptability increased between the 1 st and 4th week and was maintained at high levels after. Perceived fatigue and negative affects were variable among patients with no significant change over time (Friedman's ANOVA; Fatigue: $3.77 \pm 1.79 ; x^{2}$ [5] $=3$. 893, $p=0.56$; Negative affects: $42.85 \pm 16.66 ; \chi^{2}[5]=$ 3.084, $p=0.69$, Fig. 4). Positive affects tended to decrease over time (ANOVA, $42.3 \pm 11.2$; F $[5,45]=$ 

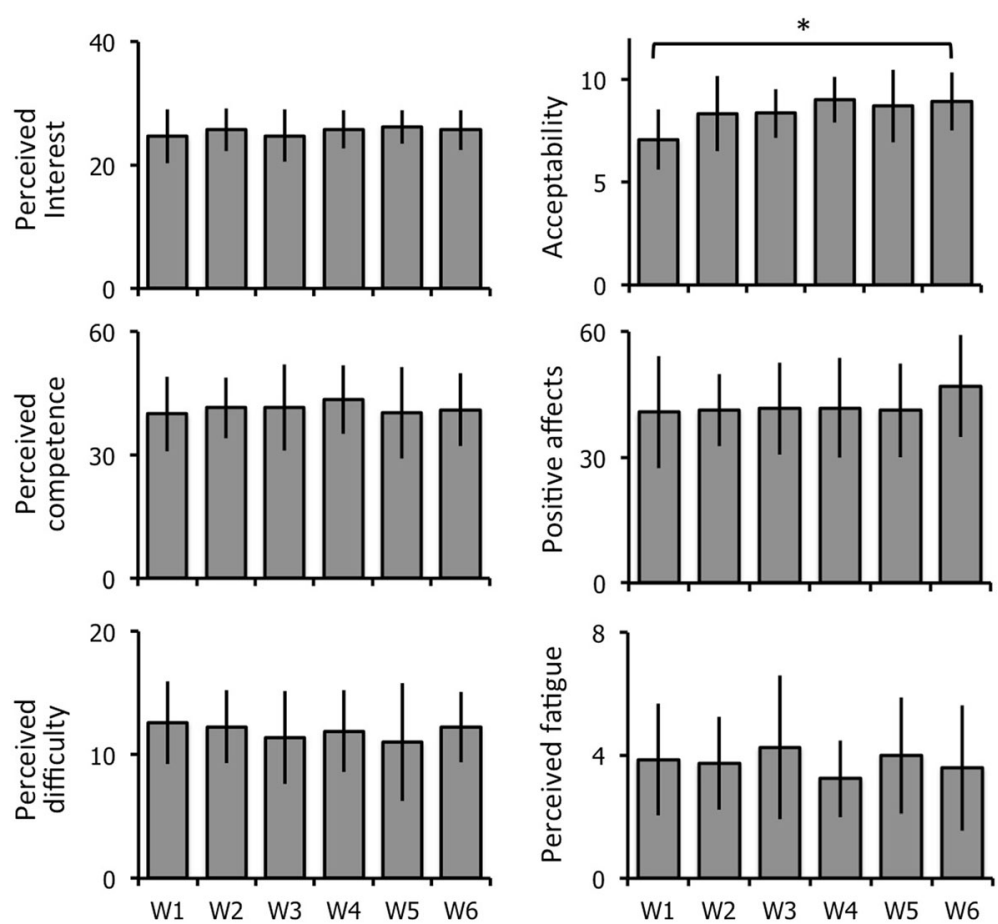

Fig. 4 Acceptability and feasibility of the 'Toap Run' videogame training in PD patients. The graphs represent the mean and standard deviation, from left to right and top to bottom, in perceived interest, perceived competence, perceived difficulty, perceived fatigue, acceptability and positive affects after the 1st session (W1) and once a week from the 2 nd to the 6th week (W2 to W6). ${ }^{*} p<0.05$ repeated measures ANOVAs

1.879, $p=0.11)$. Lastly, 7 out of 10 patients rated the game as amusing.

\section{Clinical gait and balance disorders, parkinsonian disability and quality of life}

After the 18th session, when compared to baseline, the FOG-Q, GABS-B, and axial scores significantly decreased by 39,38 and $41 \%$, respectively $(p=0.005$, 0.02 and 0.009 , respectively, Fig. 5) and ABC scale significantly increased by $35 \%(p=0.03$, Fig. 5$)$. The GABS-B and axial scores also significantly decreased by 36 and 15\%, respectively, after the 9th session ( $p=0.04$ and $p=0.008$, respectively, Fig. 5). All patients presented recurrent falls at baseline (FOG-Qitem $12>1$ ). After the 18 th session, seven of the 10 patients reported no falls ( $p=0.023$, not shown).

Parkinsonian motor disability (UPDRS part III, Fig. 5) and quality of life (PDQ-39, not shown) showed no significant change after the 18th training session relative to baseline ( $p=0.34$ and 0.13 , respectively), but the parkinsonian motor disability was significantly lower after the 9th session ( $p=0.02$, Fig. 5). The 'Activities of daily living' (UPDRS part II) score significantly decreased after the 18th session in comparison to baseline ( $p=$ 0.03, Fig. 5).
Three months later, we found no changes in the clinical scores, except a persistent significant decrease of $35 \%$ in the axial score compared to baseline ( $p=0.005$, Fig. 5$)$, and eight of ten patients reported recurrent falls again.

\section{Gait initiation recordings}

After the 18th session, the APAs phase and doublestance durations were significantly lower $(p=0.0004$ and 0.002, respectively, Fig. 6) compared to baseline, and the anteroposterior APAs displacement, step length $\left(p<10^{-4}\right.$, Fig. 6) and gait velocity $\left(p<10^{-4}\right.$, not shown) significantly increased. The mediolateral APAs displacement, step width and braking index showed no significant change (not shown). The anteroposterior APAs, step length and velocity were also significantly higher after the 9th session and 3 months later, compared to baseline (Fig. 6).

\section{Adverse events}

Three serious adverse events, unrelated to the training, were reported in three patients. Severe pneumonia occurred in one patient (P02) and a deep brain stimulation generator replacement was required in two patients (P06 and P09). The videogame training sessions were well tolerated with no specific adverse events over the course of the study. 

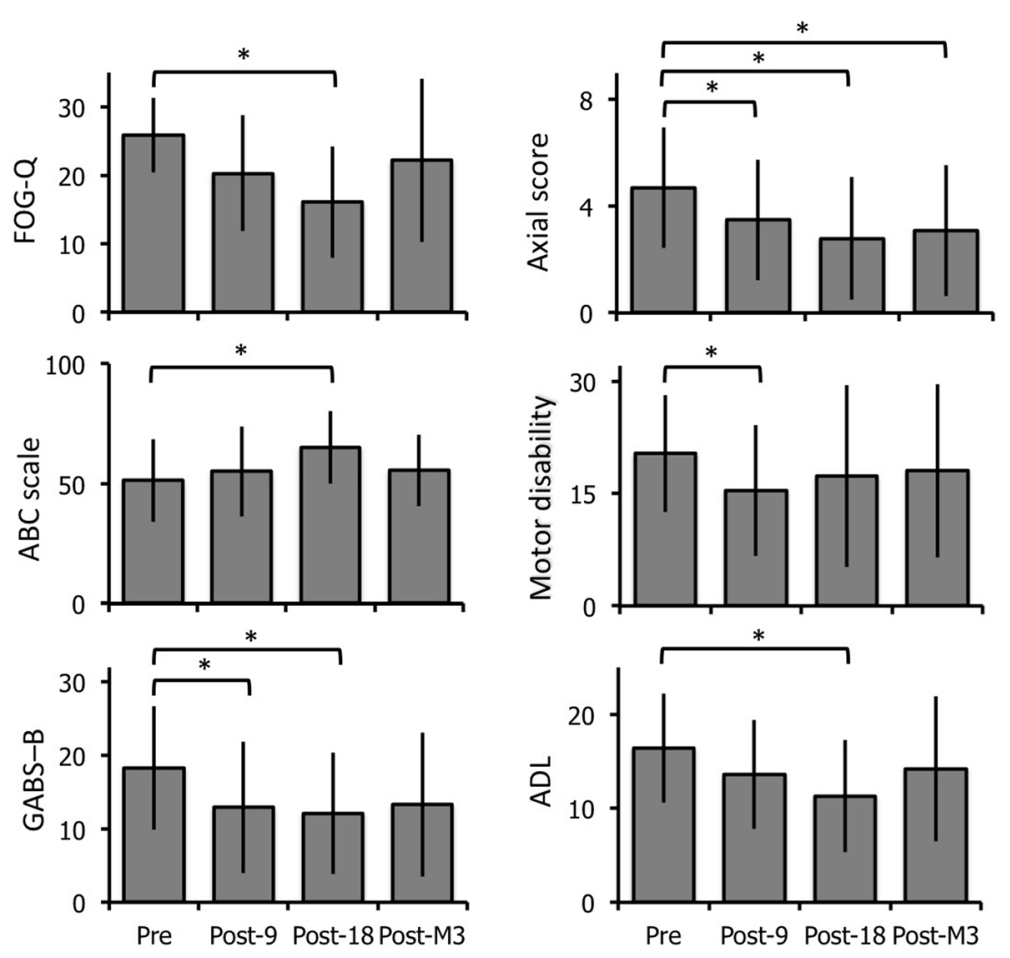

Fig. 5 Effects of the 'Toap Run' videogame training on gait and balance disorders, parkinsonian disability and quality of life in PD patients. The graphs represent the mean and standard deviation, from left to right and top to bottom, in the Freezing Of Gait Questionnaire (FOG-Q), Activities and Balance Confidence (ABC) scale, Gait And Balance scale part B (GABS-B), Axial score (UPDRS items $18+27+28+29+30$ ), parkinsonian motor disability (UPDRS part III) and activities of daily living (ADL, UPDRS part II) scores. Results were obtained before the first (Pre), after the 9th (Post-9) and 18th (Post-18) training sessions, and 3 months later (Post-M3). ${ }^{*} p<0.05$ as compared to the first (baseline) assessment

\section{Discussion}

The primary aim of this pilot study was to develop and evaluate the feasibility of a videogame rehabilitation programme using a specific customised game that targeted FOG and falls in persons with advanced and severe forms of PD. The results indicate that our videogame is feasible for use in this patient population with good overall satisfaction and motivation to use the game for training, increased performance and duration of gaming with time. All the patients gave a positive opinion about the videogame and the virtual environments proposed. Clinical assessment revealed focussed improvement in gait and balance disorders with significantly decreased severity in FOG and reduction of falls, that could be transferred to increased activities of daily living competence with better confidence in balance and increased positive affects. These clinical effects were confirmed by objective kinematic recordings showing increased anteroposterior APAs displacement, step length and velocity, which reflect better gait performance, and decreased double stance and APAs duration, which reflect better balance control $[29,38]$. The majority of these positive effects were not maintained, however, 3 months later.
This study has some limitations. These results were obtained in a small group of patients with no control group or comparative treatment, with many secondary outcomes at different time points, thus limiting the generalisation of our findings. However, from an individual point of view, all patients reported good feasibility and acceptability, with decreased severity of gait and balance disorders after training. The training was performed at the institute in the presence of the physiotherapist. This may have increased the acceptability and motivation of the patients to perform the training programme. However, 6 out of 10 patients asked to perform gaming at home at the end of the programme suggesting that our game was indeed well accepted and perceived as feasible by our patients, even in the absence of the physiotherapist. Future research with game training at home in the absence of the physiotherapist could help to decipher the respective role of the customised videogame and of the physiotherapist supervision in the effects of this rehabilitation programme. We did not specifically examine the effects of our videogame rehabilitation programme on cognition, knowing that PD patients classically presented dysexecutive syndrome. 

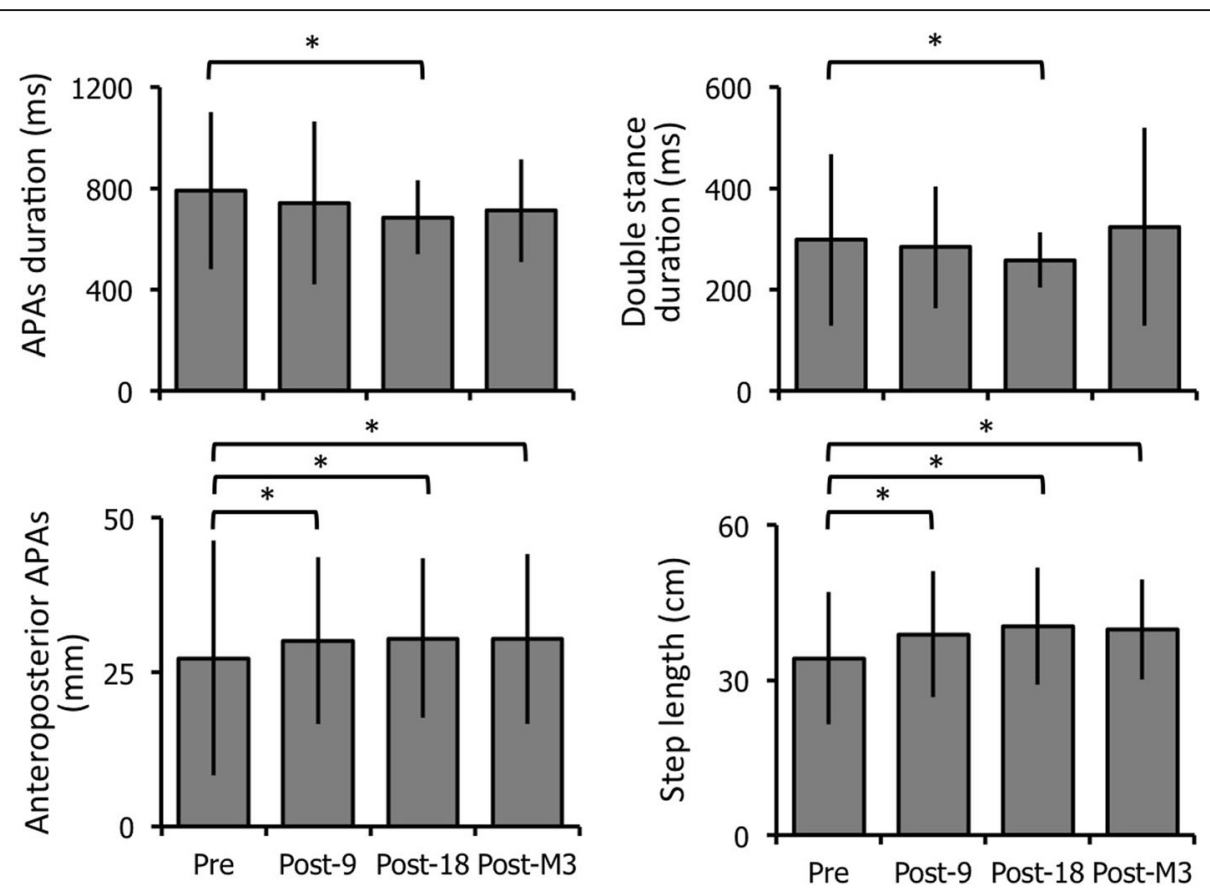

Fig. 6 Effects of the 'Toap Run' videogame training on gait initiation kinematic parameters. Each graph represents the mean and standard deviation from left to right and top to bottom of the APAs phase and double-stance durations, and anteroposterior CoP displacements during APAs and step length. Results were obtained before (Pre), after the 9th (Post-9) and 18th (Post-18) sessions, and 3 months later (Post-M3). ${ }^{*} p<$ 0.05 as compared to the first (Pre) assessment

In our study, the number of secondary outcomes with patient's interviews limited the possibility to add complete neuropsychological testing. This should be performed in future studies.

The use of commercial game training for PD rehabilitation has previously been reported in small groups of patients suffering mild to moderate forms of PD (Hoehn and Yahr score stages ranging from 1 to 3, disease duration generally less than 10 years), with good feasibility, mild improvement in parkinsonian disability, increased single-leg stance duration, dynamic balance and gait velocity, activities of daily living, quality of life and cognition [15]. Here, we tested our videogame training in severe and advanced forms of PD with resistant gait and balance disorders. These results indicate that our intervention which required high amplitude and fast movements of all four limbs combined with lateral and vertical shifts of the trunk and pelvis, with challenging and progressive exercise programme, is appropriate to treat these disabling axial motor signs. This is in line with previous studies performed in PD patients that included highly challenging balance training programmes with significant improvements in balance control and reduction in falls rate [39], or virtual reality training on a treadmill with challenging gait speed or obstacle avoidance with significant increases in gait speed and stride length $[13,40]$. The benefits were significant after at least 9 sessions of training. This reflects the need to perform at least a $40 \mathrm{~min}$ duration training session [26] and at least 8 sessions [9] to obtain clinically meaningful benefit.

The positive effects of our videogame on gait and balance performance in our PD patients could result from various physiological effects. This improvement could be related to increased corticospinal excitability of leg muscles as reported after Wii Fit training [41], thus leading to increased muscle strength [42], in particular for ankle movements with increased propulsive forces, as reflected by increased anteroposterior APAs displacement, step length and velocity, but less for hip movements with no significant change in mediolateral APAs displacement and step width $[29,38]$. Our videogame could have induced motor learning in relation to the high attentional demand induced by the need to collect the maximum of coins, negotiate obstacles, while at the same time following an external, rhythmic and progressively increasing, auditory cue [12, 23, 43]. The fact that game performance significantly increased from session to session whereas game difficulty increased (with higher visual flow velocity of the virtual environment and number of movements to perform), and that the improvement of clinical gait and balance disorders (axial-UPDRS III subscore), gait speed and step length are persistent 3 months after the last 
session are in line with this hypothesis. The use of auditory and visual cues included in the game could also have promoted faster gait speed and longer step length as classically reported in PD patients [43, 44]. Rehabilitation was performed using visual feedback (avatar movement) that could lead to neuronal plasticity as reported in sports training [45] and in balance training while learning a new skill [46]. Lastly, gaming has been shown to induce dopamine release in healthy subjects, and thus could have induced parkinsonian disability improvement [47]. However, the absence of concomitant parkinsonian motor sign improvement fails to support this hypothesis.

The positive effects on freezing of gait, balance confidence and falls observed after 18 training sessions were not maintained 3 months after the intervention. Previous studies on physiotherapy in PD patients also report a loss of benefit within weeks after discontinuation of training [8]. However, recent data suggest that the addition of virtual reality component to physical training would yield longer-term benefit [13]. The fact that PD patients included in our study had a severe and advanced form of disease could have also negatively impacted the retention effects with reduced benefit of exercise after cessation of training, as also reported in previous studies [9]. Finally, these data confirm the importance of maintaining rehabilitation over time to obtain sustained benefit.

\section{Conclusions}

These preliminary data suggest that rehabilitation based on a customised videogame to treat gait and balance disorders of PD patients is feasible, well accepted, and may be effective. The fact that the benefits of videogame training dissipate over time, as reported in other physiotherapy trials in $\mathrm{PD}$, confirms the need of sustained exercise training in PD [48]. Further larger and controlled studies are needed to confirm these data, to examine the usability by the patients themselves of such cost effective rehabilitation strategies for training at home [20] and over longer periods, but also to investigate the retention effect and the underlying processes that contribute to motor improvement.

\author{
Abbreviations \\ ABC: Activity Balance Confidence; APAs: Anticipatory postural adjustments; \\ CoM: centre of mass, FOG = freezing of gait; ${ }_{1}$ CoP: centre of foot pressure; \\ FOG-Q: freezing of gait questionnaire; GABS: gait and balance scale; \\ PD: Parkinson's disease; PDQ-39: Parkinson's Disease Questionnaire; \\ UPDRS: Unified Parkinson's disease rating scale
}

\section{Acknowledgements}

We extend our deepest thanks to all the patients who participated to this study.

\section{Funding}

This study was supported by the French National Agency for Research (ANR-13-LAB1 0003-01) and the World Innovation Competition 2014 grant for the REHAB e-NOVATION project (IA - PSMI - CMI 2014). The funding source had no involvement in the study design, collection, analysis and interpretation of data, writing of the report and decision to submit the article for publication.

\section{Availability of data and materials}

The datasets generated and/or analysed during the current study are not publicly available due to property of INSERM but are available from the corresponding author on reasonable request.

\section{Authors' contributions}

MLW, MV and PF were responsible for the conception and design of the study. MLW and PF obtained funding for the study. MLW, DN, MV, $\mathrm{CK}$ and $\mathrm{AVH}$ performed clinical investigations and acquired clinical and neurophysiological data. DN and MV performed rehabilitation training sessions. The data were analysed by DN, AVH and MLW. DN drafted the report and MLW, DN, CK, PF and AVH provided substantial input to the first draft. DN, AVH and MLW created the figures. All authors reviewed the manuscript and give final approval of the version to be submitted. The study was supervised by MLW.

\section{Authors' information}

DN is physiotherapist and PhD student; MV is physiotherapist; AVH is research engineer, specialized in kinematics data; CK is neurosurgeon, specialized in deep brain stimulation for movement disorders; PF is responsible of clinical research programme in the Genious Group; MLW is neurologist and neurophysiologist, specialized in movement disorders and gait and balance disorders.

\section{Ethics approval and consent to participate}

This study is part of clinical trial C15-12 sponsored by Inserm (ID RCB: 2015-A00277-42). It was granted approval by local Ethics Committee

(Comité de protection des personnes-lle de France V on June 2, 2015), authorised by the French authorities (ANSM, 150358B-31), and registered in a public trials registry (Trial Registration: ClinicalTrials.gov NCT02469350). All study participants gave their informed, written consent to participation, in line with French ethical guidelines.

\section{Competing interests}

Dijana Nuic, Maria Vinti, Angele Van Hamme and Carine Karachi report no conflict of interest.

Marie-Laure Welter received personal fees from Medtronic for scientific board and medical training.

Pierre Foulon is employed by Genious Systems which has no property rights on the data.

\section{Publisher's Note}

Springer Nature remains neutral with regard to jurisdictional claims in published maps and institutional affiliations.

\section{Author details \\ ${ }^{1}$ CNRS, UMR7225, Institut du Cerveau et de la Moelle Epinière, Sorbonne universités, Université Pierre et Marie Curie (UPMC) Paris P6; UMRS 1127, 75013 Paris, France. ${ }^{2}$ LabCom BRAIN e-NOVATION, Institut du Cerveau et de la Moelle épinière (ICM), 75013 Paris, France. ${ }^{3}$ Neurosurgery Department, Hôpital de la Salpêtrière, Groupe Hospitalier Pitié-Salpêtrière, APHP, 75013 Paris, France. ${ }^{4}$ GENIOUS System, 92700 Colombes, France. ${ }^{5}$ PANAM Platform, Institut du Cerveau et de la Moelle épinière, 75013 Paris, France. \\ ${ }^{6}$ Neurophysiology Department, Rouen University Hospital, Rouen-Normandie University, 76000 Rouen, France.}

Received: 19 December 2017 Accepted: 3 April 2018

Published online: 10 April 2018

\section{References}

1. Lang $A E$, Lozano AM. Parkinson's disease. Second of two parts. N Engl J Med. 1998;339(16):1130-43. 
2. Lang AE, Lozano AM. Parkinson's disease. First of two parts. N Engl J Med. 1998;339(15):1044-53.

3. Pillon B, Dubois B, Cusimano G, Bonnet AM, Lhermitte F, Agid Y. Does cognitive impairment in Parkinson's disease result from non-dopaminergic lesions? J Neurol Neurosurg Psychiatry. 1989;52(2):201-6.

4. Bloem BR, Hausdorff JM, Visser JE, Giladi N. Falls and freezing of gait in Parkinson's disease: a review of two interconnected, episodic phenomena. Mov Disord. 2004;19(8):871-84.

5. Fasano A, Aquino CC, Krauss JK, Honey CR, Bloem BR. Axial disability and deep brain stimulation in patients with Parkinson disease. Nat Rev Neurol. 2015;11(2):98-110

6. Kempster PA, Williams DR, Selikhova M, Holton J, Revesz T, Lees AJ. Patterns of levodopa response in Parkinson's disease: a clinico-pathological study. Brain. 2007;130(Pt 8):2123-8.

7. Tomlinson CL, Patel S, Meek C, Herd CP, Clarke CE, Stowe R, et al. Physiotherapy intervention in Parkinson's disease: systematic review and meta-analysis. BMJ. 2012;345:e5004.

8. Klamroth S, Steib S, Devan S, Pfeifer K. Effects of exercise therapy on postural instability in Parkinson disease: a meta-analysis. J Neurol Phys Ther. 2016;40(1):3-14.

9. Mak MK, Wong-Yu IS, Shen X, Chung CL. Long-term effects of exercise and physical therapy in people with Parkinson disease. Nat Rev Neurol. 2017; 13(11):689-703.

10. Ellis T, Boudreau JK, Deangelis TR, Brown LE, Cavanaugh JT, Earhart GM, et al. Barriers to exercise in people with Parkinson disease. Phys Ther. 2013; 93(5):628-36.

11. Mirelman A, Maidan I, Deutsch JE. Virtual reality and motor imagery: promising tools for assessment and therapy in Parkinson's disease. Mov Disord. 2013;28(11):1597-608.

12. Mirelman A, Maidan I, Herman T, Deutsch JE, Giladi N, Hausdorff JM. Virtual reality for gait training: can it induce motor learning to enhance complex walking and reduce fall risk in patients with Parkinson's disease? J Gerontol A Biol Sci Med Sci. 2011;66((2):234-40

13. Mirelman A, Rochester L, Maidan I, Del Din S, Alcock L, Nieuwhof F, et al. Addition of a non-immersive virtual reality component to treadmill training to reduce fall risk in older adults (V-TIME): a randomised controlled trial. Lancet. 2016;388(10050):1170-82.

14. Gallagher R, Damodaran H, Werner WG, Powell W, Deutsch JE. Auditory and visual cueing modulate cycling speed of older adults and persons with Parkinson's disease in a virtual cycling (V-Cycle) system. J Neuroeng Rehabil. 2016;13(1):77.

15. Dockx K, Bekkers EM, Van den Bergh V, Ginis P, Rochester L, Hausdorff JM, et al. Virtual reality for rehabilitation in Parkinson's disease. Cochrane Database Syst Rev. 2016;12:CD010760.

16. Ekker MS, Janssen S, Nonnekes J, Bloem BR, de Vries NM. Neurorehabilitation for Parkinson's disease: future perspectives for behavioural adaptation. Parkinsonism Relat Disord. 2016;22(Suppl 1): S73-7.

17. Barry G, Galna B, Rochester L. The role of exergaming in Parkinson's disease rehabilitation: a systematic review of the evidence. J Neuroeng Rehabil. 2014;11:33.

18. Esculier JF, Vaudrin J, Beriault P, Gagnon K, Tremblay LE. Home-based balance training programme using Wii Fit with balance board for Parkinsons's disease: a pilot study. J Rehabil Med. 2012;44(2):144-50.

19. Mhatre PV, Vilares I, Stibb SM, Albert MV, Pickering L, Marciniak CM, et al. Wii Fit balance board playing improves balance and gait in Parkinson disease. PM R. 2013;5(9):769-77.

20. Negrini S, Bissolotti L, Ferraris A, Noro F, Bishop MD, Villafane JH. Nintendo Wii fit for balance rehabilitation in patients with Parkinson's disease: a comparative study. J Bodyw Mov Ther. 2017;21 (1):117-23.

21. Pompeu JE, Mendes FA, Silva KG, Lobo AM, Oliveira Tde P, Zomignani AP, et al. Effect of Nintendo Wii-based motor and cognitive training on activities of daily living in patients with Parkinson's disease: a randomised clinical trial. Physiotherapy. 2012;98(3):196-204

22. Herz NB, Mehta SH, Sethi KD, Jackson P, Hall P, Morgan JC. Nintendo Wii rehabilitation ("Wii-hab") provides benefits in Parkinson's disease. Parkinsonism Relat Disord. 2013;19(11):1039-42.

23. dos Santos Mendes FA, Pompeu JE, Modenesi Lobo A, Guedes da Silva K, Oliveira Tde P, Peterson Zomignani A, et al. Motor learning, retention and transfer after virtual-reality-based training in Parkinson's disease-effect of motor and cognitive demands of games: a longitudinal, controlled clinical study. Physiotherapy. 2012;98(3):217-23.
24. Summa S, Basteris A, Betti E, Sanguineti V. Adaptive training with full-body movements to reduce bradykinesia in persons with Parkinson's disease: a pilot study. J Neuroeng Rehabil. 2015;12:16.

25. Farley BG, Koshland GF. Training BIG to move faster: the application of the speed-amplitude relation as a rehabilitation strategy for people with Parkinson's disease. Exp Brain Res. 2005;167(3):462-7.

26. Keus S, Munneke M, Graziano M, Paltamaa J, Pelosin E, Domingos J, et al. European Physiotherapy Guideline for Parkinson's Disease. the Netherlands: KNGF/Parkinson Net; 2014.

27. Hoehn MM, Yahr MD. Parkinsonism: onset, progression and mortality. Neurology. 1967;17(5):427-42.

28. Crenna P, Frigo C. A motor programme for the initiation of forwardoriented movements in humans. J Physiol. 1991;437:635-53.

29. Chastan N, Do MC, Bonneville F, Torny F, Bloch F, Westby GW, et al. Gait and balance disorders in Parkinson's disease: impaired active braking of the fall of Centre of gravity. Mov Disord. 2009;24(2):188-95.

30. Ben-Sadoun G, Sacco G, Manera V, Bourgeois J, Konig A, Foulon P, et al. Physical and cognitive stimulation using an Exergame in subjects with normal aging, mild and moderate cognitive impairment. J Alzheimers Dis. 2016;53(4):1299-314

31. Gledhill JA, Rodary C, Mahe C, Laizet C. French validation of the revised Piper Fatique Scale. Rech Soins Infirm. 2002:68:50-65.

32. Pelissolo A, Rolland JP, Perez-Diaz F, Jouvent R, Allilaire JF. Dimensional approach of emotion in psychiatry: validation of the positive and negative emotionality scale (EPN-31). Encéphale. 2007;33(3 Pt 1):256-63.

33. Thomas M, Jankovic J, Suteerawattananon M, Wankadia S, Caroline KS, Vuong KD, et al. Clinical gait and balance scale (GABS): validation and utilization. J Neurol Sci. 2004;217(1):89-99.

34. Giladi N, Shabtai H, Simon ES, Biran S, Tal J, Korczyn AD. Construction of freezing of gait questionnaire for patients with parkinsonism. Parkinsonism Relat Disord. 2000;6(3):165-70.

35. Powell LE, Myers AM. The Activities-specific Balance Confidence (ABC) scale. J Gerontol A Biol Sci Med Sci. 1995:50A(1):M28-34.

36. Martinez-Martin P, Frades Payo B. Quality of life in Parkinson's disease: validation study of the PDQ-39 Spanish version. The Grupo Centro for study of movement disorders. J Neurol. 1998;245(Suppl 1):S34-8.

37. Winter DA. Human balance and posture control during standing and walking. Gait Posture. 1995;3:193-214.

38. Burleigh-Jacobs A, Horak FB, Nutt JG, Obeso JA. Step initiation in Parkinson's disease: influence of levodopa and external sensory triggers. Mov Disord. 1997;12(2):206-15.

39. Sparrow D, DeAngelis TR, Hendron K, Thomas CA, Saint-Hilaire M, Ellis T. Highly challenging balance program reduces fall rate in Parkinson disease. $J$ Neurol Phys Ther. 2016:40(1):24-30

40. Mehrholz J, Friis R, Kugler J, Twork S, Storch A, Pohl M. Treadmill training for patients with Parkinson's disease. Cochrane Database Syst Rev. 2010;1:CD007830.

41. Esculier JF, Vaudrin J, Tremblay LE. Corticomotor excitability in Parkinson's disease during observation, imagery and imitation of action: effects of rehabilitation using wii fit and comparison to healthy controls. J Parkinsons Dis. 2014:4(1):67-75.

42. Hess JA, Woollacott M, Shivitz N. Ankle force and rate of force production increase following high intensity strength training in frail older adults. Aging Clin Exp Res. 2006;18(2):107-15.

43. Rochester L, Baker K, Hetherington V, Jones D, Willems AM, Kwakkel G, et al. Evidence for motor learning in Parkinson's disease: acquisition, automaticity and retention of cued gait performance after training with external rhythmical cues. Brain Res. 2010;1319:103-11.

44. Azulay JP, Mesure S, Amblard B, Blin O, Sangla I, Pouget J. Visual control of locomotion in Parkinson's disease. Brain. 1999:122(Pt 1):111-20.

45. Mokienko OA, Chervyakov AV, Kulikova SN, Bobrov PD, Chernikova LA, Frolov $A A$, et al. Increased motor cortex excitability during motor imagery in braincomputer interface trained subjects. Front Comput Neurosci. 2013;7:168.

46. Sehm B, Taubert M, Conde V, Weise D, Classen J, Dukart J, et al. Structural brain plasticity in Parkinson's disease induced by balance training. Neurobiol Aging. 2014;35(1):232-9.

47. Koepp MJ, Gunn RN, Lawrence AD, Cunningham VJ, Dagher A, Jones T, et al. Evidence for striatal dopamine release during a video game. Nature. 1998:393(6682):266-8.

48. Tomlinson CL, Patel S, Meek C, Clarke CE, Stowe R, Shah L, et al. Physiotherapy versus placebo or no intervention in Parkinson's disease. Cochrane Database Syst Rev. 2012;7:CD002817. 